\title{
USO EFICIENTE DA ÓRBITA DE SATÉLITES GEOESTACIONÁRIOS: OTIMIZAÇÃO DAS POSIÇÕES ORBITAIS
}

\author{
Marcelle Santiago do Nascimento e José Mauro P. Fortes
}

\begin{abstract}
Resumo - Este trabalho está relacionado ao problema do uso eficiente da órbita de satélite geoestacionário. A utilização eficiente da órbita é obtida através de um algoritmo de otimização que permite escolher posições orbitais para os satélites dos diversos sistemas considerados de modo a minimizar o arco orbital utilizado. Com esse objetivo, foi desenvolvido um modelo matemático que considerou, além de aspectos de interferência, detalhes da geometria envolvida no problema (posições orbitais dos satélites, posições das estações terrenas, apontamento de antenas, etc.). Este modelo foi utilizado na definição de um problema de otimização com restrições cuja função objetivo reflete a parcela utilizada da órbita de satélites geoestacionários. Neste problema de otimização foram consideradas restrições correspondentes aos níveis máximos permitidos de interferência (de entrada única e agregada) além das restrições de arcos orbitais, impostas por aspectos de propagação. O método matemático desenvolvido foi utilizado em situações práticas específicas de interesse conduzindo a resultados que mostraram o uso eficiente da órbita de satélites geoestacionários através de soluções que minimizam a parcela da órbita de satélites geoestacionários utilizada.
\end{abstract}

Palavras-chave: Órbita de satélites geoestacionários, análise de interferências, enfoque probabilístico, diagnóstico de ocupação orbital

\begin{abstract}
This work is related to the efficient use of the geostationary satellite orbit. It presents and describes an optimization model which chooses the best orbital position for each satellite so that the length of the used orbital arc is minimized. A mathematical model considering aspects such as interference and geometry details (satellite orbital position, earth station positions, boresights of the antennas, etc) is proposed. The model is used in the definition of a constrained optimization problem in which the objective function is defined as the length of the used orbital arc. Constraints imposed by propagation aspects (minimum elevation angles) and by the maximum allowable interference levels (aggregate and single-entry) are considered. The model is applied to specific situations involving real data, leading to the minimization of the used orbital arc.
\end{abstract}

Este trabalho teve suporte financeiro da CAPES através da Bolsa de Mestrado de Marcelle Santiago do Nascimento. Atualmente Marcelle Nascimento é engenheira da Intelig (marcelle.nascimento@inteligtelecom.com.br) e José Mauro Fortes é professor do Centro de Estudos em Telecomunicações da Pontifícia Universidade Católica do Rio de Janeiro (jmfortes@cetuc.pucrio.br)
Keywords: Geoestacionary satellite orbit, interference analysis, probabilistic approach, orbital arc evaluation

\section{INTRODUÇÃO}

A possibilidade de se fazer comunicação de longa distância utilizando um satélite que permanece parado em relação a um observador na superfície da Terra motivou fortemente o desenvolvimento das comunicações comerciais por satélite nas décadas de 60,70 e 80 . Entretanto esta possibilidade é restrita a uma única órbita, circular, no plano do equador, de raio aproximadamente igual a $42.000 \mathrm{Km}$, denominada Órbita de Satélites Geoestacionário (OSG).

Sendo a órbita de satélites geoestacionários um recurso limitado, existe motivação para a elaboração de planejamentos a priori deste recurso de modo a garantir, na prática, para todos os países, acesso eqüitativo à esta órbita. Esses planejamentos envolvem estudos, análises e modelagens de características específicas dos sistemas envolvidos, além do desenvolvimento de técnicas e algoritmos adequados a síntese do planejamento.

As técnicas aplicáveis a um determinado planejamento dependem diretamente do tipo de sistema e do tipo de serviço a ser planejado e devem levar em consideração as interferências produzidas e sofridas por cada um dos sistemas considerados. Os níveis de interferência devem ser adequados de modo a permitir a operação conjunta dos diversos sistemas. O cálculo da interferência depende basicamente das características dos sistemas envolvidos e da geometria do problema (posições orbitais dos satélites, posições das estações terrenas, direção de apontamento da antenas, etc.).

No âmbito da União Internacional de Telecomunicações, durante Conferências Mundiais de Comunicações, diversos planejamentos de serviços de comunicações por satélite foram feitos a nível internacional. Estes planejamentos tiveram início em 1977 [1] com a elaboração do Plano do Serviço de Radiodifusão por Satélite (BSS) da Europa e Ásia para a Banda Ku (11-14 GHz) ${ }^{1}$. Em 1983 [3], este planejamento foi complementado para incluir os países das Américas. Em 1988, o planejamento elaborado pela Conferência Mundial de Radiocomunicações chegou ao Serviço Fixo por Satélite para partes das bandas $C(4-6 \mathrm{GHz})$ e $K u(11-14 \mathrm{GHz})[4,6,7]$. Todos os planejamentos citados foram feitos com o auxílio de ferramentas computacionais não automatizadas que, por este motivo, requeriam siste-

${ }^{1}$ Uma revisão deste planejamento foi feita na Conferência Mundial de Comunicações de 2000 [2] 


\section{Marcelle Santiago do Nascimento e José Mauro P. Fortes Uso Eficiente da Órbita de Satélites Geoestacionários: Otimização das Posições Orbitais}

maticamente a intervenção de planejadores que, com base nas análises de interferência obtidas modificavam manualmente as posições orbitais dos satélites de modo a otimizar o planejamento. Assim, torna-se óbvio o interesse pelo desenvolvimento de ferramentas e metodologias (incluindo modelagem e algoritmos de otimização) que possam reduzir a participação direta do planejador através da síntese, mesmo que parcial, de planejamentos.

Neste trabalho foi desenvolvido um modelo matemático que permite, com base no critério de minimização do arco orbital utilizado, a determinação das posições orbitais mais adequadas para os sistemas de comunicação por satélite considerados. Esta escolha é feita respeitando as restrições de convivência impostas pelo ambiente interferente por eles produzido. A eficiência desta técnica é ilustrada através de exemplos, nos quais a técnica é aplicada a situações específicas envolvendo o serviço fixo por satélite.

Na Seção 2, o problema a ser examinado é definido. Sua geometria é caracterizada, e são definidos os parâmetros utilizados pelo modelo de otimização proposto, apresentado na Seção 3. Na Seção 4 são apresentados resultados obtidos com a aplicação do modelo proposto a situações específicas de interesse. Finalmente, na Seção 5, as principais conclusões obtidas a partir do trabalho desenvolvido são apresentadas.

\section{MODELO MATEMÁTICO}

O principal requisito que deve ser considerado quando se fala em planejamento da órbita de satélites geoestacionários é a convivência dos sistemas de comunicações por satélite, ou seja, operação conjunta dos diversos sistemas sem o comprometimento do desempenho de cada um deles por efeito de interferência. A medida da quantidade de interferência existente entre dois sistemas é geralmente baseada na, assim chamada, Razão Portadora-Interferência. Esta razão pode ser calculada em dois níveis: considerando-se a componente de interferência devido a um único sistema (Razão Portadora-Interferência de Entrada Única) ou considerandose o agregado das interferências geradas pelos diversos sistemas (Razão Portadora-Interferência Agregada).

O cálculo da interferência produzida ou experimentada pelos diversos sistemas que compartilham a órbita de satélites geoestacionários e que operam em uma mesma faixa de frequiências depende das características técnicas dos sistemas e, em grande parte, da geometria envolvida no problema, ou seja, dos espaçamentos orbitais relativos entre os satélites, das localizações das estações terrenas e das direções para as quais suas antenas apontam. Sendo assim, a geometria e a notação utilizada constituem pontos importantes para a definição do modelo matemático que será desenvolvido. A Figura 1 ilustra a geometria utilizada nas definições das razões Portadora-Interferência de entrada única e agregada. Nesta figura estão indicados dois satélites: um satélite interferente $S_{I}$ e um satélite vítima $S_{V}$. As quantidades assinaladas superiormente com o sinal " $"$ " descrevem as características do sistema interferente enquanto que as quantidades sem o sinal " " estão associadas ao sistema vítima. Nesta mesma figura, $P_{1}$ e $P_{1}^{\prime}$ representam as potências nos termi- nais das antenas das estações terrenas de transmissão, $g_{1}$ e $g_{1}^{\prime}$ os ganhos das antenas das estações terrenas de transmissão e $g_{2}$ e $g_{2}^{\prime}$ os ganhos das antenas receptoras dos satélites. As quantidades $P_{3}$ e $P_{3}^{\prime}$ representam as potências nos terminais das antenas de transmissão dos satélites, $g_{3}$ e $g_{3}^{\prime}$ os ganhos das antenas de transmissão dos satélites e $g_{4}$ e $g_{4}^{\prime}$ os ganhos das antenas das estações terrenas receptoras. Além disso, $I_{d}$ e $I_{u}$ indicam as interferências no lance de descida e no lance de subida, respectivamente. Note que as interferências $I_{d}, I_{u}$ assim como os ângulos $\theta, \eta, \rho, \xi$, identificados na Figura 1, dependem das posições dos satélites e das estações terrenas. Os ângulos $\theta, \eta, \rho, \xi$ são definidos mais adiante.

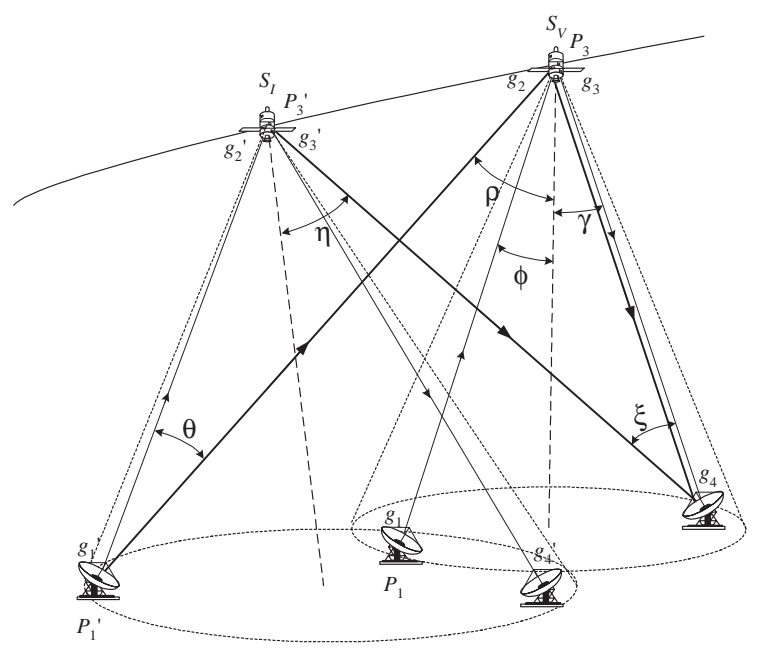

Figura 1. Geometria envolvida no cálculo da Razão Portadora Interferente.

Considerando-se os enlaces interferente e interferido ilustrados na Figura 1 a razão $C / I$ de entrada única no terminal da antena de recepção do satélite da rede interferida $S_{V}$ (Razão Portadora-Interferente no lance de subida) se escreve [8]

$$
\left(\frac{C}{I}\right)_{u p}=\frac{\frac{P_{1} g_{1}(0) g_{2}(\phi)}{\ell_{s u}}}{\frac{P_{1}^{\prime} g_{1}^{\prime}(\theta) g_{2}(\rho)}{\ell_{s u^{\prime}}}}=\frac{P_{1} g_{1}(0) g_{2}(\phi) \ell_{s u^{\prime}}}{P_{1}^{\prime} g_{1}^{\prime}(\theta) g_{2}(\rho) \ell_{s u}}
$$

onde $g_{1}^{\prime}(\theta)$ é o ganho da antena da estação terrena transmissora do sistema interferente numa direção que forma um ângulo $\theta$ com a direção de apontamento da antena, $g_{1}(0)$ é o ganho máximo da antena da estação terrena transmissora do sistema interferido, $g_{2}(\phi)$ é o ganho da antena receptora do satélite da rede interferida numa direção que forma um ângulo $\phi$ com a direção de apontamento do feixe e $g_{2}(\rho)$ é o ganho da antena receptora do satélite da rede interferida numa direção que forma um ângulo $\rho$ com a direção de apontamento do feixe.

De maneira análoga, a razão $C / I$ de entrada única no terminal da antena da estação terrena receptora da rede interferida devido apenas à interferência no lance de descida (Razão Portadora-Interferência no lance de descida) se escreve [8]

$$
\left(\frac{C}{I}\right)_{\text {down }}=\frac{\frac{P_{3} g_{4}(0) g_{3}(\gamma)}{\ell_{s d}}}{\frac{P_{3}^{\prime} g_{3}^{\prime}(\eta) g_{4}(\xi)}{\ell_{s d^{\prime}}}}=\frac{P_{3} g_{3}(\gamma) g_{4}(0) \ell_{s d^{\prime}}}{P_{3}^{\prime} g_{3}^{\prime}(\eta) g_{4}(\xi) \ell_{s d}}
$$


onde $g_{3}^{\prime}(\eta)$ é o ganho da antena transmissora do satélite interferente numa direção que forma um ângulo $\eta$ com a direção de apontamento do feixe, $g_{4}(\xi)$ é o ganho da antena da estação terrena receptora numa direção que forma um ângulo $\xi$ com a direção de apontamento da antena, $g_{4}(0)$ é o ganho máximo da antena da estação terrena receptora e $g_{3}(\gamma)$ é o ganho da antena transmissora do satélite da rede interferida numa direção que forma um ângulo $\gamma$ com a direção de apontamento.

Em (1) e (2) $\ell_{s u}$ e $\ell_{s d}$ representam as perdas de espaço livre associadas aos percursos do sinal desejado nos lances de subida e descida, respectivamente. De maneira análoga, as quantidades $\ell_{s u^{\prime}}$ e $\ell_{s d^{\prime}}$ representam as perdas de espaço livre associadas aos percursos da interferência nos lances de subida e descida, respectivamente. Estas perdas são calculadas utilizando-se a expressão [8]

$$
\ell=\left(\frac{4 \pi d \cdot f_{u}}{3}\right)^{2} \cdot 10^{8}
$$

onde $d$ é a distância percorrida pelo sinal, dada em $\mathrm{km}$ e $f_{u}$ é a frequiência utilizada na transmissão, dada em GHz.

A partir de (1) e (2) obtém-se a Razão PortadoraInterferência total no terminal da antena da estação terrena receptora da rede interferida, dada por

$$
\left(\frac{C}{I}\right)_{\text {total }}=\left[\left(\frac{C}{I}\right)_{u p}^{-1}+\left(\frac{C}{I}\right)_{\text {down }}^{-1}\right]^{-1}
$$

ou seja,

$$
\left(\frac{C}{I}\right)_{\text {total }}=\left[\frac{P_{1}^{\prime} g_{1}^{\prime}(\theta) g_{2}(\rho) \ell_{s u}}{P_{1} g_{1}(0) g_{2}(\phi) \ell_{s u^{\prime}}}+\frac{P_{3}^{\prime} g_{3}^{\prime}(\eta) g_{4}(\xi) \ell_{s d}}{P_{3} g_{3}(\gamma) g_{4}(0) \ell_{s d^{\prime}}}\right]^{-1}
$$

A expressão em (4) fornece a razão entre a potência da portadora desejada e da interferência total (interferência nos lances de subida e descida) causada por um único sistema interferente.

Considerando-se um ambiente interferente envolvendo $n$ sistemas operando na mesma faixa de freqüências, a Razão Portadora-Interferência Agregada, correspondente à interferência em um determinado sistema ( $i$, por exemplo), devido às transmissões dos demais sistemas, é dada por

$$
\left(\frac{C}{I}\right)_{a g_{i}}=\left(\sum_{\substack{j=1 \\ j \neq i}}^{n}\left(\frac{C}{I}\right)_{i j}^{-1}\right)^{-1} \quad ; i=1, \ldots, n
$$

onde

$$
\left(\frac{C}{I}\right)_{i j}=\frac{C_{i}}{I_{j}}
$$

é a Razão Portadora - Interferência de Entrada Única correspondente à parcela de interferência produzida pelo sistema $j$ nos terminais da antena da estação terrena receptora do sistema $i$, determinada utilizando-se (4). Em (6), $C_{i}$ denota a potência do sinal desejado correspondente ao sistema $i$ e $I_{j}$ representa a potência interferente total (nos lances de subida e descida) devido ao sistema $j$.
O cálculo das razões Portadora-Interferência em (4) e (5) requer o conhecimento dos diagramas de radiação das estações terrenas e de suas localizações geográficas, dos diagramas de radiação das antenas dos satélites de suas posições orbitais. Cada um desses pontos será comentado a seguir.

Os diagramas de radiação adotados neste trabalho se baseiam em recomendações do Setor de Radiocomunicações da União Internacional Telecomunicações (UIT-R), mais especificamente, no Apêndice 30B do Regulamento de Radicomunicações [5, 6]. Para o diagrama de radiação da antena da estação terrena, considerou-se o caso particular de antenas com padrão de lóbulo lateral melhorado $(29-25 \log (\Phi))$. Esse diagrama é definido por

$$
G(\Phi)= \begin{cases}G_{\max }-\frac{1}{400}\left(\frac{D}{\lambda} \Phi\right)^{2} & ; \quad 0<\Phi<\Phi_{m} \\ G_{1} & ; \quad \Phi_{m} \leq \Phi<\Phi_{r} \\ 29-25 \log (\Phi) & ; \quad \Phi_{r} \leq \Phi<36.3^{\circ} \\ -10 & ; \quad 36.3^{\circ} \leq \Phi<180^{\circ}\end{cases}
$$

onde $G_{\max }$ e $G_{1}$ representam, respectivamente, o ganho máximo e o ganho do primeiro lóbulo lateral da antena, dados por

$$
G_{\max }=10 \log \left[\eta\left(\frac{\pi D}{\lambda}\right)^{2}\right]
$$

$\mathrm{e}$

$$
G_{1}=-1+15 \log \left(\frac{D}{\lambda}\right)
$$

Em (7), o ângulo $\Phi$ correponde ao desvio angular em relação à direção de apontamento da antena (em graus) e $G(\Phi)$ é o ganho da antena na direção do ângulo $\Phi$, expresso em dB. Os ângulos $\Phi_{m}$ e $\Phi_{r}$, expressos em graus, são dados por

$$
\begin{aligned}
\Phi_{m} & =\frac{20 \lambda}{D} \sqrt{G_{\max }-G_{1}} \\
\Phi_{r} & =15.85\left(\frac{D}{\lambda}\right)^{-0.6}
\end{aligned}
$$

Em (7)-(11), $D$ é o diâmetro da antena (feito igual a $3 \mathrm{~m}$ ) e $\lambda$ é o comprimento de onda correspondente à faixa de freqüências considerada $(\lambda=0.025 \mathrm{~m}$ para a Banda $\mathrm{Ku}$ 12GHz). A eficiência $\eta$ da antena foi considerada igual a 0.6. Esses valores, quando aplicados a (8)-(11), conduzi$\operatorname{ram}$ a $G_{\max }=49.3 \mathrm{~dB}, G_{1}=30.188 \mathrm{~dB}, \Phi_{m}=0.73^{\circ} \mathrm{e}$ $\Phi_{r}=0.90^{\circ}$.

Para definir o diagrama de radiação da antena do satélite deve-se, primeiramente, ressaltar que foram considerados satélites que possuem feixes com formato elíptico. Isso significa que, num plano perpendicular à direção de apontamento da antena de transmissão do satélite, os pontos de meia potência (onde a potência sofre um decréscimo de $3 \mathrm{~dB}$ em relação a direção de apontamento - contorno de $-3 \mathrm{~dB}$ ) descrevem uma elipse, conforme ilustrado na Figura 2. É interessante ressaltar que a escolha dos vetores $\mathbf{m}$ e $\mathbf{r}$ mostrados na Figura 2 é muito importante. Eles servem de base para a orientação da elípse (a orientação da elípse é definida pelo ângulo entre o seu eixo maior e uma reta paralela ao plano do equador). Um desses vetores, $\mathbf{m}$ por exemplo, deve ter sua direção paralela ao plano do equador. A projeção sobre a Terra do contorno de $-3 \mathrm{~dB}$, que delimita a área de cobertura do satélite, apresenta-se com uma distorção devido aos 


\section{Marcelle Santiago do Nascimento e José Mauro P. Fortes Uso Eficiente da Órbita de Satélites Geoestacionários: Otimização das Posições Orbitais}

efeitos da curvatura da Terra. A expressão que define o diagrama de radiação da antena do satélite depende da razão $\Psi$ entre os ângulos $\Phi$ e $\Phi_{0}$, definidos na Figura 2, sendo dada por [4]

$$
G(\Psi)= \begin{cases}G_{\max }-12(\Psi)^{2} & ; 0<\Psi \leq 1.45 \\ G_{\max }-(22+20 \log (\Psi)) & ; 1.45<\Psi \leq 15 \\ G_{\max }-(22+20 \log (15)) & ; \Psi>15\end{cases}
$$

onde $G_{\max }$, corresponde ao ganho na direção de apontamento da antena do satélite, que depende dos ângulos $\phi_{01} \mathrm{e}$ $\phi_{02}$ que determinam o eixo maior e menor da elipse que define a área de serviço do satélite, sendo dado por

$$
G_{\max }=44.45-10 \log \left(\phi_{01} \cdot \phi_{02}\right)
$$

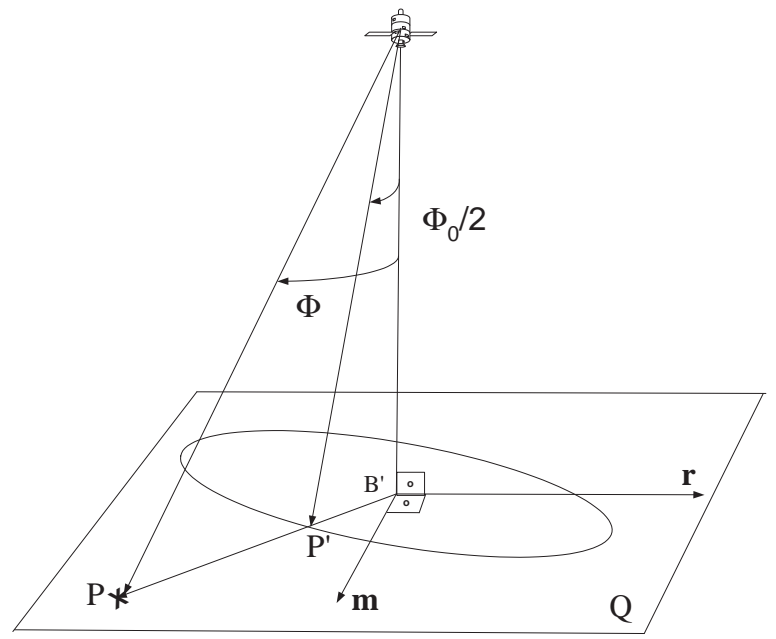

Figura 2. Ângulos de referência para os diagramas de radiação das antenas dos satélites.

O critério utilizado para escolha da localização das estações terrenas de transmissão e recepção das redes de comunicação por satélites envolvidas consiste em escolher a posição mais desfavorável em termos da quantidade de interferência gerada (no caso da estação terrena transmissorainterferente) e da quantidade de interferência sofrida (no caso da estação terrena receptora-interferida).

A posição orbital de um satélite geoestacionário é definida pela longitude de sua localização. A escolha da posição orbital de um determinado satélite deve ser feita considerandose algumas limitações. Estas limitações são, geralmente, devidas à restrições de propagação (eg. limite inferior de ângulo de elevação) e à restrições operacionais (eg. distribuição do tráfego a ser atendido, existência de um sistema na posição desejada, níveis máximos aceitáveis de interferência de entrada única e agregada, etc). A parcela da órbita que respeita essas limitações é denominada arco de serviço do satélite considerado.

\section{MODELO MATEMÁTICO PARA OTIMIZAÇÃO DO USO DA ÓRBITA}

Dado um conjunto de $n$ redes $\left\{R_{1}, R_{2}, R_{3}, \ldots R_{n}\right\}$, seja $i$ $(i=1, \ldots, n)$ o índice que caracteriza a rede interferida e $j$ $(j=1, \ldots, n ; i \neq j)$ o índice que caracteriza a rede interferente. Considere a situação ilustrada na Figura 1, onde $n$ redes de comunicações por satélite compartilham o arco orbital. Deseja-se especificar as posições orbitais dos satélites dessas redes de modo a minimizar a parcela de arco orbital utilizada garantindo, ao mesmo tempo, que os níveis de interferência que afetam cada uma dessas redes estejam abaixo dos níveis máximos permitidos e que a posição orbital pertença ao arco de serviço a ela associado.

Seja $x_{i}$ a posição orbital do satélite $S_{i}$ da $i$-ésima rede de comunicações por satélite. As posições orbitais dos satélites das redes envolvidas são então descritas por um vetor $n$ dimensional $\mathbf{x}=\left(\begin{array}{llll}x_{1} & x_{2} & \ldots & x_{n}\end{array}\right)^{T}$.

$\mathrm{O}$ arco orbital utilizado pelos satélites dos $n$ sistemas se escreve

$$
\mathcal{P}=f(\mathbf{x})=\max \left(x_{1}, \ldots, x_{n}\right)-\min \left(x_{1}, \ldots, x_{n}\right)
$$

onde as funções $\max ($ ) e $\min ($ ) indicam, respectivamente, o maior e o menor de seus argumentos.

A minimização da função objetivo em (13) deve ser feita de forma que as seguintes restrições sejam satisfeitas:

- Restrições associadas aos níveis de Razão Portadora Interferência Total de entrada única $(n(n-1)$ restrições).

$$
\begin{aligned}
h_{i j}(\mathbf{x})=\left(\frac{C}{I}\right)_{i j} \geq L_{s e} ; \quad & i=1, \ldots, n \\
j & =1, \ldots, n, j \neq i
\end{aligned}
$$

onde $(C / I)_{i j}$ é a Razão Portadora Interferência de entrada única do sistema $j$ no sistema $i$, conforme equação (4) e $L_{s e}$ é o nível máximo permitido para esta razão.

- Restrições associadas aos níveis de Razão Portadora Interferência agregada sobre cada sistema ( $n$ restrições).

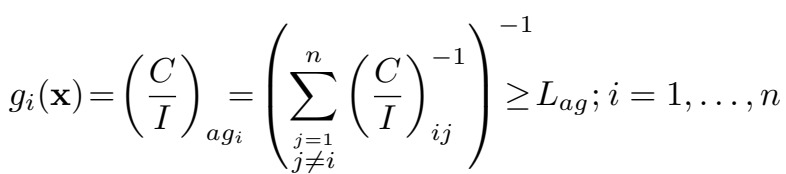

onde onde $(C / I)_{i}$ é a Razão Portadora Interferência de entrada agregada sobre o sistema $i$ e $L_{a g}$ é o nível máximo permitido para a esta razão.

- Restrições associadas aos arcos de serviço dos sistemas ( $n$ restrições).

$$
a_{i} \leq x_{i} \leq b_{i}, \quad i=1, \ldots n
$$

onde o intervalo $\left[a_{i}, b_{i}\right]$ é o arco de serviço que cada sistema deve respeitar.

Assim o problema a ser resolvido consiste em determinar o valor de $\mathbf{x}$ que minimiza a função objetivo (13) e satisfaz as restrições em (14), (15) e (16). 
Solucionar de maneira genérica esse problema de otimização pode se tornar uma tarefa bastante difícil, uma vez que o número de variáveis envolvidas, não linearmente relacionadas, é muito grande. A complexidade envolvida na busca de soluções para esse problema geral pode, em algumas situações específicas, ser reduzida. Este é o caso particular em que a ordem dos satélites na órbita é pré-estabelecida. Esta pré-fixação pode ser feita sem perda de generalidade, uma vez que a solução ótima do problema geral (sem a pré-fixação da ordem) pode ser obtida considerando-se todas as possíveis ordenações, comparando-se as soluções a elas associadas e escolhendo a que fornece o menor valor da função objetivo.

Desta forma, seja $\tilde{\mathbf{x}}=\left(\begin{array}{llll}\tilde{x}_{1} & \tilde{x}_{2} & \ldots & \tilde{x}_{n}\end{array}\right)^{T}$ o vetor de posições orbitais ordenadas, tal que $\tilde{x}_{1}<\tilde{x}_{2}<\ldots<\tilde{x}_{n}$. A parcela do arco orbital utilizada se escreve

$$
\tilde{\mathcal{P}}=f(\tilde{\mathbf{x}})=\tilde{x}_{n}-\tilde{x}_{1}
$$

Um novo problema é então definido, onde deseja-se determinar o valor $\tilde{\mathbf{x}}^{*}$ do vetor $\tilde{\mathbf{x}}$ que minimiza a função objetivo em (17) satisfazendo às restrições em (14), (15) e (16) e às $n-1$ restrições adicionais impostas pela pré-ordenação das posições orbitais dos satélites, dadas por

$$
\tilde{x}_{i-1}<\tilde{x}_{i} ; i=1, \ldots, n
$$

Seria interessante que o espaço dos valores de $\tilde{\mathbf{x}}$ que satisfazem às restrições (14), (15), (16) e (18) fosse convexo, o que garantiria a convergência do resultado para um mínimo global. Note que as restrições em (16) e (18) definem regiões limitadas por hiperplanos. Se considerarmos que a razão $\left(\frac{C}{I}\right)_{i j}$ se mantém constante quando o espaçamento entre os satélites não varia (aproximação), as restrições em (14) conduzirão também a regiões limitadas por hiperplanos. Assim, a região definida pelas restrições (16), (18) e (14) pode ser considerada convexa. (interseção de regiões limitadas por hiperplanos).

No que diz respeito às restrições em (15) nada pode-se afirmar a priori. Entretanto, testes efetuados durante a realização deste trabalho [9] indicaram que, nos exemplos de aplicação realizados, diferentes valores iniciais para $\tilde{\mathbf{x}}_{0}$, conduziram a um mesmo valor de $\tilde{\mathbf{x}}^{*}$.

Um algoritmo original que gera o conjunto de ordenações possíveis (aquelas que satisfazem às restrições de arco orbital e interferência) foi desenvolvido em [9].

\section{RESULTADOS NUMÉRICOS}

O modelo matemático desenvolvido na Seção 3 é aqui utilizado para otimizar as posições orbitais dos satélites de sistemas que compartilham a órbita de satélites geoestacionários. Na obtenção dos resultados, foi utilizada a técnica numérica de otimização conhecida como Sequential Uncostrained Minimization Technique - SUMIT [10], na qual o problema de otimização com restrições é solucionado resolvendo-se uma seqüência de problemas de otimização sem restrições. A escolha desta técnica foi motivada apenas pela alta complexidade das expressões utilizadas no cálculo do gradiente e da matriz Hessiana associados ao problema (ver [9]). A utilização dessas expressões em técnicas de otimização sem restrições é bem mais simples. Três exemplos, representando situações específicas, são apresentados. Os parâmetros dos sistemas considerados nesses exemplos são idênticos aos utilizados no Plano do Serviço Fixo por Satélite elaborado União Internacional de Telecomunicações em 1988 [6]. Os valores mínimos permissíveis de $(C / I)_{i j}$ e $(C / I)_{i}$ foram tomados, respectivamente, iguais a

$$
L_{s e}=30 \mathrm{~dB}
$$

e

$$
L_{a g}=26 \mathrm{~dB}
$$

No primeiro exemplo, o modelo desenvolvido é utilizado para otimizar as posições orbitais de 10 satélites de sistemas domésticos que compartilham a órbita de satélites geoestacionários cobrindo países da Europa.

Os parâmetros dos sistemas considerados neste exemplo estão apresentados na Tabela 1 . Nesta tabela, $\theta_{B}$ e $\phi_{B}$ representam, respectivamente, a longitude e a latitude da direção de apontamento da antena de transmissão do satélite, $\phi_{01}$ e $\phi_{02}$ definem o eixo maior e menor do contorno de $-3 \mathrm{~dB}$ do feixe elíptico do satélite, $\gamma$ representa o ângulo de orientação da elipse (azimute do eixo maior), $a_{i}$ e $b_{i}$ representam os limites inferior e superior do arco de serviço associado ao $i$-ésimo sistema.

As áreas de cobertura associadas aos sistemas considerados neste exemplo estão ilustradas na Figura 3. Estas áreas de cobertura são definidas pela interseção entre a elípse que caracteriza o contorno de $-3 \mathrm{~dB}$ da antena transmissora do satélite e a superfície da Terra. A solução ótima encontrada, que corresponde à menor ocupação da órbita, é dada por

$$
\begin{aligned}
& \mathbf{x}^{*}=\left(-22,0^{\circ}-17,28^{\circ}-12,93^{\circ}-20,6^{\circ}-6,5^{\circ}\right. \\
& \left.4,1^{\circ} \quad 10,1^{\circ} \quad 37,92^{\circ} \quad 35,0^{\circ} \quad 39,48^{\circ}\right)^{T}
\end{aligned}
$$

sendo a ordenação a ela associada

$$
x_{1}, x_{4}, x_{2}, x_{3}, x_{5}, x_{6}, x_{7}, x_{9}, x_{8}, x_{10}
$$

A convergência do processo de otimização correspondente a esta ordenação é ilustrada na Figura 4. As curvas do lado esquerdo desta figura ilustram a evolução das posições orbitais dos satélites envolvidos, ao longo do processo de otimização. Estão também indicados na figura o arco orbital $\Delta_{i}$ utilizado pelos satélites no início do processo (Iteração 1) e o arco orbital $\Delta_{f}$ utilizado pelos satélites ao final do processo (Iteração 10). As linhas verticais no lado direito da Figura 4 mostram, a título de referência, os arcos de serviço associados a cada um dos sistemas. Em cada um desses arcos de serviço, a posição orbital final de sistema a ele associado é indicada por um " $\times$ ". Nota-se que, para a ordenação $x_{1}, x_{4}, x_{2}, x_{3}, x_{5}, x_{6}, x_{7}, x_{9}, x_{8}, x_{10}$, o processo de otimização foi iniciado com uma utilização orbital $\Delta_{i}=99.80^{\circ}$ e atingiu a utilização orbital mínima 
Tabela 1. Parâmetros dos sistemas considerados no primeiro exemplo

\begin{tabular}{||c|c|c|c|c|c|c|c|c|c|c||}
\hline \hline & País & $\begin{array}{c}\theta_{B} \\
\text { [graus] }\end{array}$ & $\begin{array}{c}\phi_{B} \\
\text { [graus] }\end{array}$ & $\begin{array}{c}\phi_{01} \\
\text { [graus] }\end{array}$ & $\begin{array}{c}\phi_{02} \\
\text { [graus] }\end{array}$ & $\begin{array}{c}\gamma \\
\text { [graus] }\end{array}$ & $\begin{array}{c}\text { e.i.r. } p_{E T} \\
{[\mathrm{~dB}(\mathrm{~W} / \mathrm{hz})]}\end{array}$ & $\begin{array}{c}\text { e.i.r. } p_{S A T} \\
{[\mathrm{~dB}(\mathrm{~W} / \mathrm{hz})]}\end{array}$ & $\begin{array}{c}\mathrm{a}_{i} \\
\text { [graus] }\end{array}$ & $\begin{array}{c}\mathrm{b}_{i} \\
\text { [graus] }\end{array}$ \\
\hline \hline 1 & Portugal & $-8,0$ & 39,7 & 0,8 & 0,8 & 60,0 & $-8,1$ & $-28,1$ & $-55,0$ & $-22,0$ \\
2 & Espanha & $-3,0$ & 39,9 & 2,1 & 1,2 & 8,0 & $-1,8$ & $-27,8$ & $-50,0$ & $-11,0$ \\
3 & França & 3,1 & 45,9 & 2,1 & 1,1 & 168,0 & $-0,2$ & $-26,3$ & $-30,0$ & 9,0 \\
4 & Irlanda & 54,3 & 33,0 & 3,7 & 1,5 & 143,0 & 2,0 & $-27,5$ & $-30,0$ & 9,0 \\
5 & Suíça & 8,2 & 46,5 & 0,8 & 0,8 & 90,0 & $-9,3$ & $-29,4$ & $-20,0$ & 9,0 \\
6 & Itália & 11,3 & 40,9 & 2,1 & 1,0 & 141,0 & $-0,7$ & $-26,4$ & 4,0 & 34,0 \\
7 & Bulgaria & 25,6 & 42,8 & 0,8 & 0,8 & 90,0 & $-7,9$ & $-28,1$ & 10,0 & 42,0 \\
8 & Alemanha & $-4,1$ & 53,9 & 1,6 & 1,0 & 178,0 & $-4,3$ & $-28,0$ & 10,0 & 42,0 \\
9 & Hungria & 19,4 & 47,4 & 0,8 & 0,8 & 90,0 & $-7,9$ & $-28,1$ & 35,0 & 60,0 \\
10 & Romênia & 25,0 & 46,3 & 1,5 & 1,0 & 178,0 & $-4,3$ & $-28,0$ & 35,0 & 65,0 \\
\hline \hline
\end{tabular}

$\Delta_{f}=61.50^{\circ}$. Observa-se ainda que a solução encontrada satisfaz às restrições de arco de serviço.

Os valores de $(C / I)_{i j}$ e $(C / I)_{i}$ correspondentes à solução ótima encontrada estão apresentados na Tabela 2.

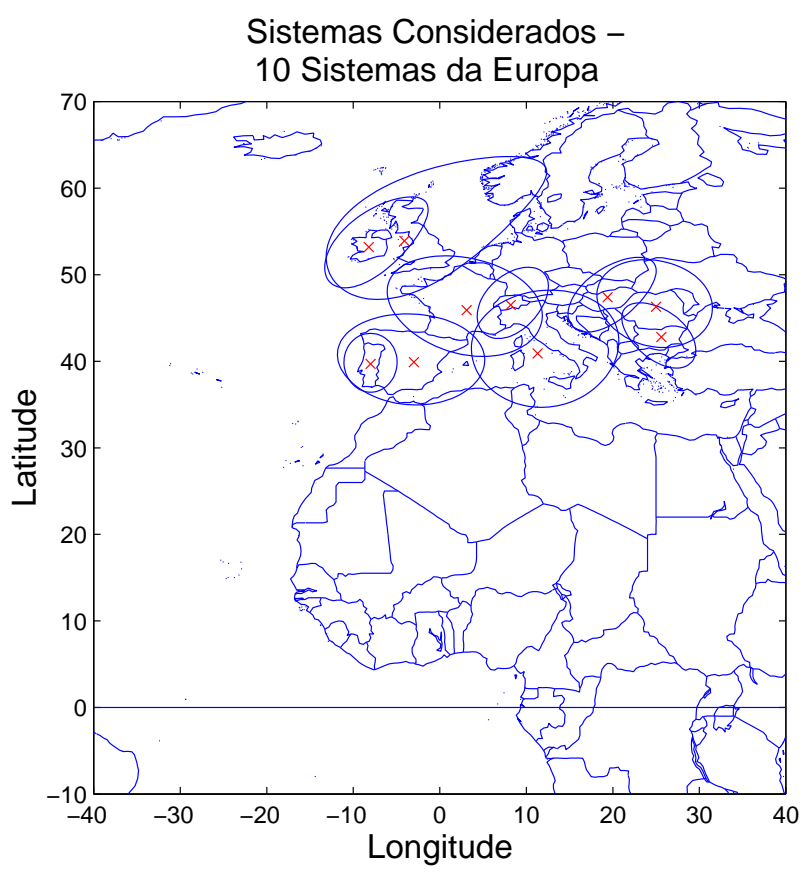

Figura 3. Coberturas dos sistemas considerados no primeiro exemplo (Europa).

Observe que alguns dos valores de razão portadorainterferência de entrada única que aparecem nesta tabela são iguais ao valor mínimo admissível $L_{s e}$, mostrando que, conforme esperado, a solução encontrada está na fronteira imposta pelo conjunto das restrições consideradas.

No segundo exemplo, o modelo desenvolvido na Seção 3 é utilizado para otimizar as posições orbitais de 10 satélites de sistemas domésticos que compartilham a órbita de satélites geoestacionários cobrindo países da América Latina.

Os parâmetros dos sistemas considerados neste exemplo estão apresentados na Tabela 3 , onde $\theta_{B}, \phi_{B}, \phi_{01}, \phi_{02}$, $\gamma$, e.i.r.p $p_{E T}$, e.i.r.p $p_{S A T}, a_{i}$ e $b_{i}$ têm significados idênticos àqueles definidos anteriormente em conexão com a Tabela 1.

As áreas de cobertura associadas aos sistemas considera-

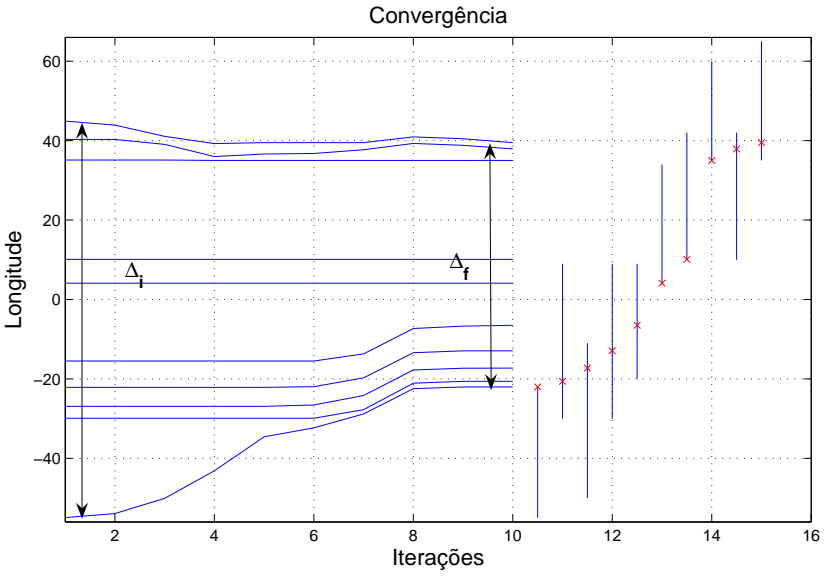

Figura 4. Convergência do processo de otimização para a ordenação que conduziu à utilização do menor arco orbital no primeiro exemplo.

dos neste exemplo estão ilustradas na Figura 5. A solução ótima encontrada, que corresponde à menor ocupação da órbita, é dada por

$$
\begin{aligned}
& \mathbf{x}^{*}=\left(-63,18^{\circ}-61,46^{\circ}-43,67^{\circ}-58,55^{\circ}-39,95^{\circ}\right. \\
& \left.-54,23^{\circ}-41,89^{\circ}-48,9^{\circ}-35,43^{\circ}-31,59^{\circ}\right)^{T}
\end{aligned}
$$

sendo a ordenação a ela associada

$$
x_{1}, x_{2}, x_{4}, x_{6}, x_{8}, x_{3}, x_{7}, x_{5}, x_{9}, x_{10}
$$

A convergência do processo de otimização correspondente a esta ordenação é ilustrada na Figura 6. Note nesta figura que, para esta ordenação, o processo de otimização foi iniciado com uma utilização orbital $\Delta_{i}=61,90^{\circ}$ e atingiu a utilização orbital mínima $\Delta_{f}=31,59^{\circ}$. Observa-se ainda que a solução encontrada satisfaz às restrições de arco de serviço.

Os valores de $(C / I)_{i j}$ e $(C / I)_{i}$ correspondentes a solução ótima encontrada estão apresentados na Tabela 4.

Observe que, como no primeiro exemplo, alguns dos valores de razão portadora-interferência de entrada única que aparecem nesta tabela são iguais ao valor mínimo admissível $L_{s e}$. Além disso, alguns dos valores de razão 
Tabela 2. Razões Portadora-Interferência de entrada única $(C / I)_{i j}$ Portadora-Interferência agregada $(C / I)_{i}$ em dB, correpondentes à solução ótima do primeiro exemplo.

\begin{tabular}{|c|c|c|c|c|c|c|c|c|c|c|c|}
\hline \multirow{2}{*}{$\begin{array}{c}\text { sistema } \\
\text { vítima }\end{array}$} & \multicolumn{10}{|c|}{ sistema interferente - $(C / I)_{i j}[\mathrm{~dB}]$} & \multirow{2}{*}{$\begin{array}{c}(C / I)_{i} \\
{[\mathrm{~dB}]} \\
\end{array}$} \\
\hline & 1 & 4 & 2 & 3 & 5 & 6 & 7 & 9 & 8 & 10 & \\
\hline 1 & 0 & 35,3 & 30,1 & 38,4 & 59,9 & 56,6 & 59,9 & 63,1 & 61 & 61,4 & 28,6 \\
\hline 4 & 32,5 & 0 & 34,9 & 36,3 & 56,6 & 52,1 & 57,7 & 62,5 & 50,0 & 58,8 & 29,8 \\
\hline 2 & 36,7 & 44,0 & 0 & 30,0 & 46,8 & 49,4 & 60,4 & 64,5 & 59,7 & 66,7 & 29,5 \\
\hline 3 & 48,1 & 48,4 & 33,3 & 0 & 42,5 & 46,6 & 61,5 & 64,9 & 56,1 & 63,3 & 32,9 \\
\hline 5 & 57,2 & 56,6 & 37,7 & 30,5 & 0 & 34,9 & 55,6 & 60,2 & 49,7 & 60,0 & 28,6 \\
\hline 6 & 65,7 & 64,0 & 52,0 & 46,1 & 46,5 & 0 & 49,2 & 65,3 & 57,4 & 64,7 & 42,1 \\
\hline 7 & 62,2 & 62,6 & 55,9 & 54,1 & 60,5 & 42,5 & 0 & 54,9 & 56,4 & 51,3 & 41,3 \\
\hline 9 & 63,2 & 65,2 & 57,8 & 55,5 & 62,8 & 56,3 & 52,8 & 0 & 34,3 & 30,0 & 29,2 \\
\hline 8 & 64,6 & 56,8 & 57,6 & 50,9 & 56,8 & 52,8 & 57,9 & 38,0 & 0 & 30,9 & 30,4 \\
\hline 10 & 65,2 & 65,3 & 63,7 & 57,5 & 66,6 & 59,5 & 53,0 & 33,7 & 30,3 & 0 & 28,7 \\
\hline
\end{tabular}

Tabela 3. Parâmetros dos sistemas considerados no segundo exemplo

\begin{tabular}{|c|c|c|c|c|c|c|c|c|c|c|}
\hline & País & $\begin{array}{c}\theta_{B} \\
\text { [graus] } \\
\end{array}$ & $\begin{array}{l}\phi_{B} \\
\text { [graus] } \\
\end{array}$ & $\begin{array}{l}\phi_{01} \\
\text { [eraus] }\end{array}$ & $\begin{array}{l}\phi_{02} \\
\text { [graus] }\end{array}$ & $\begin{array}{c}\gamma \\
\text { [graus] }\end{array}$ & $\begin{array}{c}\text { e.i.r. } p_{E T} \\
{[\mathrm{~dB}(\mathrm{~W} / \mathrm{h} z \mathrm{z})]} \\
\end{array}$ & $\begin{array}{c}\text { e.i.r.p. } p_{S A T} \\
{[\mathrm{daB}(\mathrm{Whzz})]} \\
\end{array}$ & $\begin{array}{c}\mathrm{a}_{i} \\
\text { [graus] }\end{array}$ & $\begin{array}{c}\mathrm{b}_{i} \\
\text { [graus] }\end{array}$ \\
\hline 1 & Venezuela & $-66,4$ & 6,8 & 2,8 & 2,1 & 142,0 & 5,8 & $-22,7$ & $-92,0$ & - \\
\hline 2 & Argentina & $-62,0$ & $-33,6$ & 4,8 & 2,9 & 93,0 & 10,3 & $-21,9$ & $-85,0$ & $-49,1$ \\
\hline 3 & Bolívia & $-64,4$ & $-17,1$ & 2,7 & 1,7 & 129,0 & 5,2 & $-22,5$ & $-78,0$ & $-42,1$ \\
\hline 4 & Brasil 1 & $-62,6$ & $-6,0$ & 4,1 & 4,0 & 43,0 & 10,7 & $-22,4$ & $-75,0$ & $-49,1$ \\
\hline 5 & Cuba & $-79,5$ & 21,0 & 2,0 & 1,0 & 172,0 & 1,0 & $-24,6$ & $-65,0$ & $-38,1$ \\
\hline 6 & Paraguai & $-58,7$ & $-23,1$ & 1,5 & 1,3 & 116,0 & 1,6 & $-22,8$ & $-60,0$ & $-25,0$ \\
\hline 7 & Guiana & $-59,2$ & 4,7 & 1,4 & 1,0 & 94,0 & $-0,5$ & $-22,8$ & $-49,0$ & $-21,0$ \\
\hline 8 & Uruguai & $-56,3$ & $-33,7$ & 1,1 & 1,0 & 58,0 & $-5,6$ & $-27,7$ & $-48,9$ & $-19,0$ \\
\hline 9 & Brasil 2 & $-45,4$ & $-6,3$ & 4,6 & 4,1 & 152,0 & 11,3 & $-22,4$ & $-42,0$ & $-10,0$ \\
\hline 10 & Brasil 3 & $-50,0$ & $-20,9$ & 4,3 & 3,0 & 60,0 & 9,8 & $-22,2$ & $-38,0$ & 0 \\
\hline
\end{tabular}

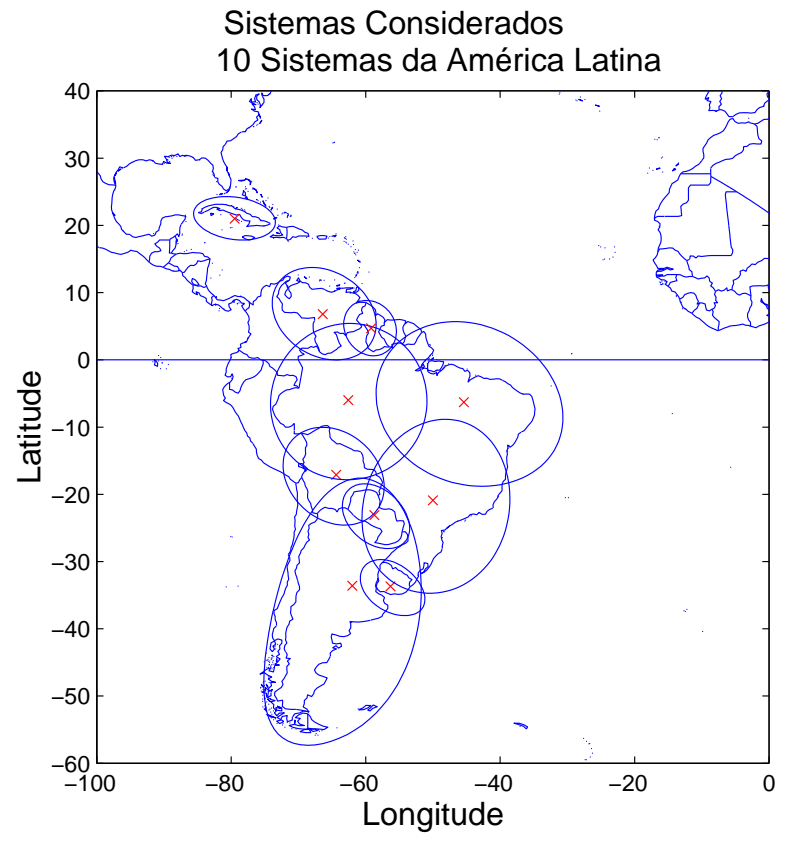

Figura 5. Coberturas dos sistemas considerados no primeiro exemplo (América do Sul).

portadora-interferência agregada são iguais ao valor mínimo admissível $L_{a g}$, mostrando que, conforme se poderia esperar,

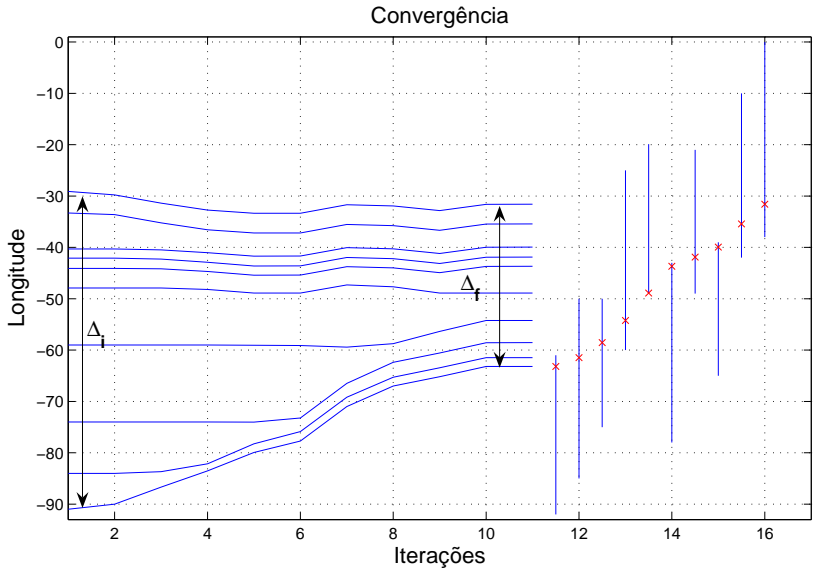

Figura 6. Convergência do processo de otimização para a ordenação que conduziu à utilização do menor arco orbital no segundo exemplo.

a solução encontrada está na fronteira imposta pelo conjunto das restrições consideradas.

Um terceiro exemplo considera uma situação mais realista na qual é feita uma otimização local do uso da órbita de satélites geoestacionários. Neste caso, são também considerados no problema sistemas cujas posições orbitais não estão sendo otimizadas mas que se encontram na vizinhança das 
Tabela 4. Razões Portadora-Interferência de entrada única $(C / I)_{i j}$ Portadora-Interferência agregada $(C / I)_{i}$ em dB, correpondentes à solução ótima do segundo exemplo.

\begin{tabular}{||c||c|c|c|c|c|c|c|c|c|c||c||}
\hline \hline \multicolumn{1}{|c||}{$\begin{array}{c}\text { sistema } \\
\text { vítima }\end{array}$} & \multicolumn{10}{c||}{ sistema interferente $-(C / I)_{i j}[\mathrm{~dB}]$} & \multicolumn{1}{c||}{$(C / I)_{i}$} \\
\cline { 2 - 12 } & 1 & 2 & 4 & 6 & 8 & 3 & 7 & 5 & 9 & 10 & {$[\mathrm{~dB}]$} \\
\hline \hline 1 & 0 & 30,0 & 30,0 & 51,7 & 56,9 & 63,1 & 51,7 & 63,3 & 54,2 & 64,4 & 27,0 \\
2 & 34,6 & 0 & 30,6 & 42,1 & 55,9 & 49,9 & 57,5 & 55,4 & 57,4 & 53,4 & 30,0 \\
4 & 35,2 & 30,5 & 0 & 40,2 & 54,9 & 47,6 & 49,9 & 58,9 & 50,3 & 54,6 & 29,6 \\
6 & 46,6 & 31,8 & 30,0 & 0 & 47,0 & 39,1 & 54,1 & 50,8 & 49,0 & 45,2 & 27,5 \\
8 & 40,2 & 34,2 & 33,0 & 35,5 & 0 & 34,5 & 38,4 & 36,2 & 36,3 & 34,8 & 26,0 \\
3 & 62,5 & 44,0 & 42,0 & 43,5 & 50,6 & 0 & 35,9 & 39,5 & 41,6 & 41,2 & 32,1 \\
7 & 45,2 & 46,4 & 38,2 & 52,8 & 48,7 & 30,0 & 0 & 33,3 & 31,6 & 42,5 & 26,3 \\
5 & 56,3 & 43,5 & 46,5 & 48,5 & 45,6 & 32,7 & 32,4 & 0 & 30,0 & 35,6 & 26,0 \\
9 & 59,9 & 57,9 & 50,8 & 59,8 & 58,8 & 47,8 & 43,7 & 43,1 & 0 & 31,3 & 30,9 \\
10 & 68,5 & 52,3 & 53,7 & 54,5 & 55,5 & 46,0 & 53,0 & 47,0 & 30,0 & 0 & 30,0 \\
\hline \hline
\end{tabular}

Tabela 5. Parâmetros dos sistemas adicionais considerados no terceiro exemplo

\begin{tabular}{||c|c|c|c|c|c|c|c|c|c||}
\hline \hline & País & $\begin{array}{c}\text { Pos. } \\
\text { Orbital }\end{array}$ & $\begin{array}{c}\theta_{B} \\
\text { [graus] }\end{array}$ & $\begin{array}{c}\phi_{B} \\
\text { [graus] }\end{array}$ & $\begin{array}{c}\phi_{01} \\
\text { [graus] }\end{array}$ & $\begin{array}{c}\phi_{02} \\
\text { [graus] }\end{array}$ & $\begin{array}{c}\gamma \\
\text { [graus] }\end{array}$ & $\begin{array}{c}\text { e.i.r.p. } E T \\
{[\mathrm{~dB}(\mathrm{~W} / \mathrm{hz})]}\end{array}$ & $\begin{array}{c}\text { e.i.r.p. } S A T \\
{[\mathrm{~dB}(\mathrm{~W} / \mathrm{hz})]}\end{array}$ \\
\hline \hline 11 & Suriname & $-23,0$ & $-55,6$ & 3,9 & 1,0 & 0,9 & 37,0 & $-2,7$ & $-23,2$ \\
12 & Chile & $-70,0$ & $-82,6$ & $-32,8$ & 8,1 & 6,1 & 155,0 & 9,9 & $-28,4$ \\
\hline \hline
\end{tabular}

posições orbitais a serem otimizadas e que, por este motivo, impõem restrições adicionais. Neste exemplo, dois outros sistemas, cujas características técnicas são apresentadas na Tabela 5, são adicionados àqueles considerados no exemplo anterior (América Latina). As posições orbitais desses dois sistemas permanecem fixas durante o processo de otimização e, conforme já mencionado, embora esses sistemas adicionais não façam parte do conjunto de sistemas cujas posições orbitais estão sendo otimizadas, eles impõem restrições adicionais de interferência. As posições orbitais desses dois sistemas permanecem fixas durante o processo de otimização. A solução ótima encontrada neste terceiro exemplo, que corresponde à menor ocupação da órbita, é dada por

$$
\begin{aligned}
x^{*} & =\left(-60,93^{\circ}-62.76^{\circ}-53.77^{\circ}-65.56^{\circ}-56.62^{\circ}\right. \\
& \left.-40.72^{\circ}-42.94^{\circ}-48.26^{\circ}-37.47^{\circ}-33.64^{\circ}\right)^{T}
\end{aligned}
$$

sendo a ordenação a ela associada

$$
x_{4}, x_{2}, x_{1}, x_{5}, x_{3}, x_{8}, x_{7}, x_{6}, x_{9}, x_{10}
$$

A convergência do processo de otimização correspondente a esta ordenação é ilustrada na Figura 7. Note nesta figura que, para esta ordenação, o processo de otimização foi iniciado com uma utilização orbital $\Delta_{i}=33,0^{\circ}$ e atingiu a utilização orbital mínima $\Delta_{f}=31,92^{\circ}$. Observa-se ainda que a solução encontrada satisfaz às restrições de arco de serviço. Observa-se que, neste exemplo, a parcela de arco orbital utilizada é maior do que a parcela de arco orbital utilizada pelos sistemas no exemplo anterior. Isso era de se esperar, uma vez que o terceiro exemplo possui restrições adicionais. Os valores de $(C / I)_{i j}$ e $(C / I)_{i}$ correspondentes a solução ótima encontrada estão apresentados na Tabela 6. Observe que, como no segundo exemplo, alguns dos valores de razão portadora-interferência de entrada única que aparecem nesta tabela são iguais ao valor mínimo admissível $L_{s e}$ e alguns dos valores de razão portadora-interferência agregada

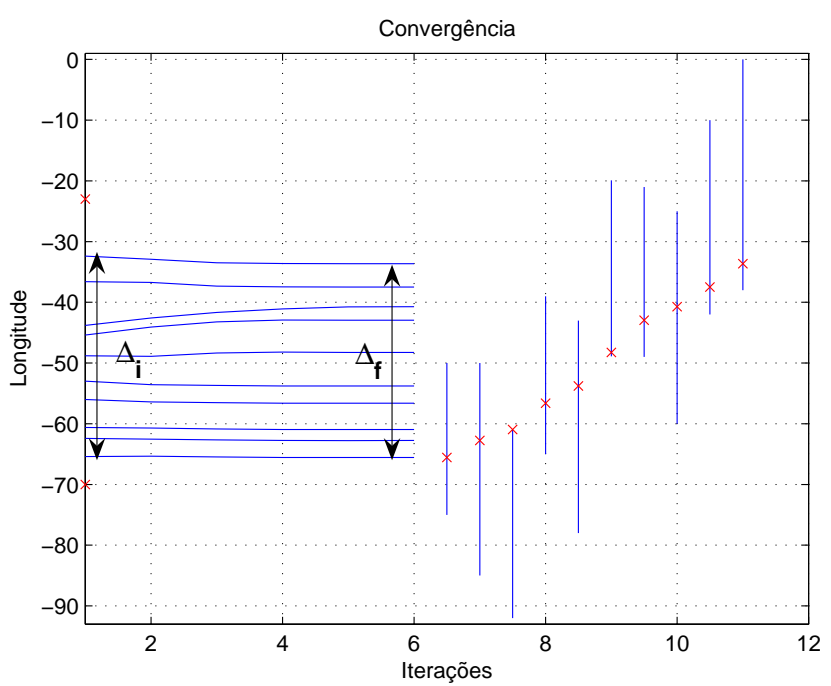

Figura 7. Convergência do processo de otimização para a ordenação que conduziu à utilização do menor arco orbital no terceiro exemplo.

são iguais ao valor mínimo admissível $L_{a g}$. Isto significa que, conforme ocorreu nos exemplos anteriores, a solução encontrada neste caso está novamente na fronteira imposta pelo conjunto das restrições consideradas.

\section{CONCLUSÃO}

Este trabalho abordou o problema do uso eficiente do recurso da órbita de satélites geoestacionários. Foi desenvolvido um modelo matemático que considerou além dos aspectos de interferência, detalhes da geometria envolvida no problema (posições orbitais dos satélites, posições das estações terrenas, direção de apontamento de antenas, etc). 
Tabela 6. Razões Portadora-Interferência de entrada única $(C / I)_{i j}$ Portadora-Interferência agregada $(C / I)_{i}$ em dB, correspondentes à solução ótima do terceiro exemplo.

\begin{tabular}{|c|c|c|c|c|c|c|c|c|c|c|c|c|c|}
\hline \multirow{2}{*}{$\begin{array}{c}\text { sistema } \\
\text { vítima }\end{array}$} & \multicolumn{12}{|c|}{ sistema interferente - $(C / I)_{i j}$ [dB] } & \multirow{2}{*}{$\begin{array}{c}(C / I)_{2} \\
{[\mathrm{~dB}]}\end{array}$} \\
\hline & 12 & 4 & 2 & 1 & 5 & 3 & 8 & 7 & 6 & 9 & 10 & 11 & \\
\hline 12 & 0 & 30,8 & 36,8 & 41,8 & 45,8 & 47,9 & 54,2 & 50,6 & 53,5 & 54,1 & 54,8 & 66,3 & 29,31 \\
\hline 4 & 44,3 & 0 & 30,0 & 35,2 & 50,7 & 45,0 & 61,2 & 53,2 & 59,0 & 52,3 & 55,9 & 70,9 & 29,01 \\
\hline 2 & 49,9 & 30,1 & 0 & 35,2 & 41,6 & 42,4 & 57,5 & 57,5 & 53,9 & 57,3 & 53,1 & 69,7 & 29,18 \\
\hline 1 & 50,1 & 30,0 & 30,7 & 0 & 45,2 & 52,2 & 55,5 & 49,9 & 60,6 & 52,5 & 62,8 & 68,6 & 27,17 \\
\hline 5 & 47,1 & 38,6 & 30,0 & 38,4 & 0 & 30,0 & 45,4 & 54,3 & 50,2 & 45,9 & 46,7 & 61,9 & 26,10 \\
\hline 3 & 55,8 & 39,4 & 36,7 & 51,6 & 36,5 & 0 & 51,2 & 55,5 & 45,9 & 50,2 & 46,9 & 63,7 & 31,99 \\
\hline 8 & 45,8 & 39,3 & 35,8 & 38,8 & 35,7 & 35,0 & 0 & 35,4 & 39,7 & 33,9 & 33,1 & 46,5 & 26,00 \\
\hline 7 & 52,5 & 41,5 & 46,5 & 43,4 & 54,8 & 49,6 & 45,7 & 0 & 34,1 & 30,0 & 41,3 & 49,4 & 27,94 \\
\hline 6 & 56,7 & 48,8 & 43,5 & 55,5 & 52,1 & 41,3 & 51,2 & 35,4 & 0 & 30,0 & 32,2 & 50,3 & 27,11 \\
\hline 9 & 68,1 & 52,9 & 57,8 & 58,2 & 58,6 & 56,3 & 56,4 & 42,2 & 40,9 & 0 & 31,2 & 62,5 & 30,95 \\
\hline 10 & 67,2 & 55,2 & 52,0 & 66,9 & 57,8 & 51,7 & 53,8 & 51,9 & 41,4 & 30,0 & 0 & 57,1 & 29,88 \\
\hline 11 & 62,2 & 53,7 & 53,1 & 57,2 & 56,9 & 52,0 & 51,1 & 43,8 & 43,3 & 44,7 & 40,9 & 0 & 36,33 \\
\hline
\end{tabular}

Este modelo matemático foi utilizado na definição de um problema de otimização com restrição cuja função objetivo está diretamente associada à parcela do arco orbital utilizada para acomodar os sistemas envolvidos. Observou-se que a complexidade da função objetivo escolhida é bastante reduzida se a ordenação dos satélites dos diversos sistemas na órbita é fixada. Assim, optou-se por resolver o problema de otimização com restrição para cada uma das possíveis ordenações dos sistemas na órbita e comparar as soluções encontradas.

O modelo proposto foi aplicado a três exemplos. O primeiro deles considerou sistemas domésticos cobrindo países da Europa, o segundo considerou sistemas domésticos cobrindo países das Américas e o terceiro exemplo ilustrou a possibilidade de utilização do modelo proposto numa otimização localizada (otimizção local) da órbita. Neste caso, além das restrições de níveis de interferência de entrada única e agregada referente aos sistemas considerados na otimização, foram consideradas as restrições adicionais de níveis de interferência de entrada única e agregada para os sistemas que possuem posições orbitais na vizinhança das extremidades do arco orbital a ser minimizado.

A eficiência e a utilização da órbita foi medida em termos do arco orbital necessário para acomodar os sistemas considerados sem desrespeitar as restrições de interferência e arco de serviço requerido por cada sistema. Os resultados numéricos mostraram que a metodologia proposta pode também ser aplicada a regiões limitadas da órbita (otimização localizada) o que corresponde a uma aplicação importante para atualização (melhora) de planejamentos já efetuados.

\section{REFERÊNCIAS}

[1] Planing of the Broadcasting Satellite Service in Regions 1 and 3, Tecnical Basis for planning, Final Acts - World Broadcasting - Satellite Administrative Radio Conference, Geneva, 1977.

[2] Revision of the Broadcasting Satellite Service im Regions 1 and 3, Technical Basis for planing, Final Acts - World Radiocommunication Conference, Stambul, May, 2000.
[3] Regional Administrative Radio Conference for the planing of the BroadCasting-Satellite Service in Region 2, Technical Basis for Planing, Final Acts - Geneva, 1983.

[4] Technical Basis for the World Administrative Radio Conference on the Use of the Geostationary-Satellite Orbit and the Planing of space Services Utilizing it, Report of the CCIR, July 1984.

[5] Radio Regulations, International Telecommunication Union, Geneva, 2003.

[6] World administrative Radio Conference on the Use of the Geostationary-Satellite Orbit and the Planning on the space Services Utilizing it, Final Acts, Outubro 1988, Geneva Apêndice 30B.

[7] CCIR Report to the Second Session of the World Administrative Radio Conference on the Use of the GeostationarySatellite Orbit and the planing of Space Services Utilizing it, Technical Report of the CCIR, July 1988.

[8] Handbook on Satellite Communications, International Telecommunication Union, John Wiley \& Sons Inc., 2002.

[9] Nascimento, Marcelle S., Uso Eficiente da Órbita de Satélites Geoestacionários: Otimização das Posições Orbitais, Dissertação de Mestrado, Departamento de Engenharia Elétrica, PUC-Rio, abril 2005.

[10] A. V. Fiacco \& G. P. McCormick, Nonlinear Programming: Sequential Unconstrained Minimization Techniques, John Wiley, 1968.

Marcelle Santiago do Nascimento graduou-se em Engenharia de Telecomunicações pela Universidade Federal Fluminense em dezembro de 2002. Obteve o grau de Mestre em Ciências de Engenharia Elétrica em 2005 pela Pontifícia Universidade Católica do Rio de Janeiro. Atualmente é Engenheira da Intelig Telecomunicaçoes LTDA. Suas áreas de interesse incluem comunicação via satélite e transmissão digital.

José Mauro P. Fortes graduou-se em Engenharia Elétrica (Telecomunicações) em 1973 pela Pontifícia Universidade Católica do Rio de Janeiro (PUC-Rio). Em 1976 obteve, na mesma universidade, o título de Mestre em Ciências de Engenharia Elétrica. Obteve ainda os títulos de MSc e PhD pela Universidade de Stanford, Califórnia - EUA, em 1978 e 1980, respectivamente. Retornou à PUC-Rio em Junho de 1980, onde é atualmente Professor Associado do Departamento de Engenharia Elétrica, lotado no Centro de Estudos em Telecomunicações da universidade. Durante o ano 
de 1992, enquanto em licença sabática, foi pesquisador do General Electric Research and Development Center, em Schenectady EUA, atuando no Ultrasound Research Group. O Professor Fortes publicou vários artigos em periódicos e conferências nacionais e internacionais. Participou de diversos projetos de pesquisa e prestou consultoria a diversas empresas privadas e agências governamentais. Durante 13 anos foi Vice-Presidente do Grupo de Estudos 4 (Servi- ço Fixo por Satélite) do Setor de Radicomunicações da União Internacional de Telecomunicações (ITU), em Genebra. Foi ainda Presidente da Sociedade Brasileira de Telecomunicações de Março de 1996 a Fevereiro de 2000, sendo atualmente Sócio Sênior da sociedade. Seus interesses de pesquisa incluem transmissão via satélite, teoria das comunicações, teoria de estimação e transmissão digital. 